\title{
Dosukoi-Tap: The Virtual Paper Sumo Game
}

\author{
Yuta Nakagawa, Kota Tsukamoto, and Yasuyuki Kono \\ Kwansei Gakuin University, 2-1 Gakuen, Sanda city, Hyogo, Japan \\ \{nakagawa717a, tsukamoto, kono\}@kwansei.ac.jp
}

\begin{abstract}
We have developed a virtual paper sumo game, "Dosukoi-Tap", a Japanese traditional game using paper figures. A player taps on his/her ownside of the sumo ring board. He/she lets his/her own wrestler rush and fight with its opponent. Our system simulates the feature of actual paper sumo and has solved some of the problems of actual one by employing multi-finger tracking and pressure-sensitive device.
\end{abstract}

Keywords. virtual game, multi finger tracking, pressure sensitive.

\section{Introduction}

Paper sumo game is one of the popular tabletop games in Japan. This game follows sumo rules by using paper figures. Two players play this game with each sumo wrestler figure made of paper as depicted in Fig.1. Each player puts his/her own paper wrestler facing each other on the sumo ring board that is typically made of a paper box. The player taps on his/her own-side of the sumo ring board and lets his/her own wrestler rush and fight with its opponent. The match is decided when either wrestler has been out from the sumo ring or lost balance and fallen down. Because of the simplicity, many of Japanese children have played the game. However, it cannot be played well when paper wrestlers don't collide or fall down before the game heated.

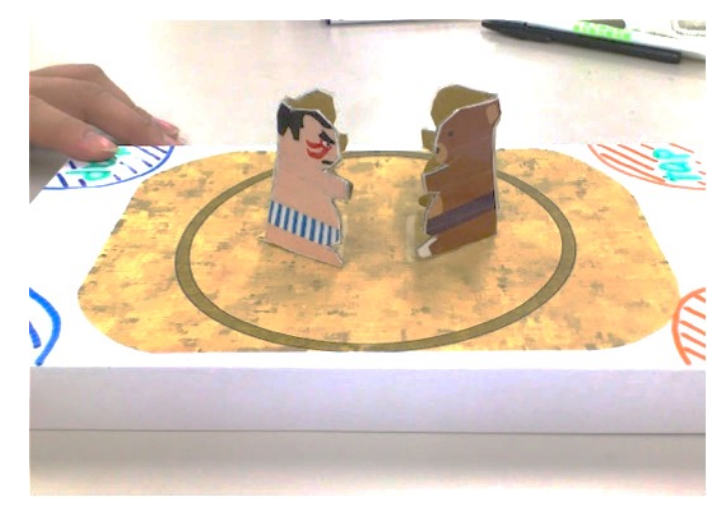

Fig. 1. paper sumo game 
We propose the virtual paper sumo game "Dosukoi-Tap" which solves following problems of the actual paper sumo game:

- The wrestler will fall down easily.

- The wrestlers do not push each other.

- It is difficult to find out a strategy.

This simulation is reproduced by the system employing the multi-finger tracking and pressure-sensitive device.

\section{Dosukoi-Tap}

Dosukoi-Tap basically simulates paper sumo employing a multi-finger tracking and pressure-sensitive touch pad as an input device. This game solves many of the paper sumo problems while inheriting the pleasure of the actual paper sumo and is more interesting than actual one on the following features:

- The wrestler does not accidently fall down.

- The wrestlers basically face and push each other.

- Original swipe operations and the strategies are introduced.

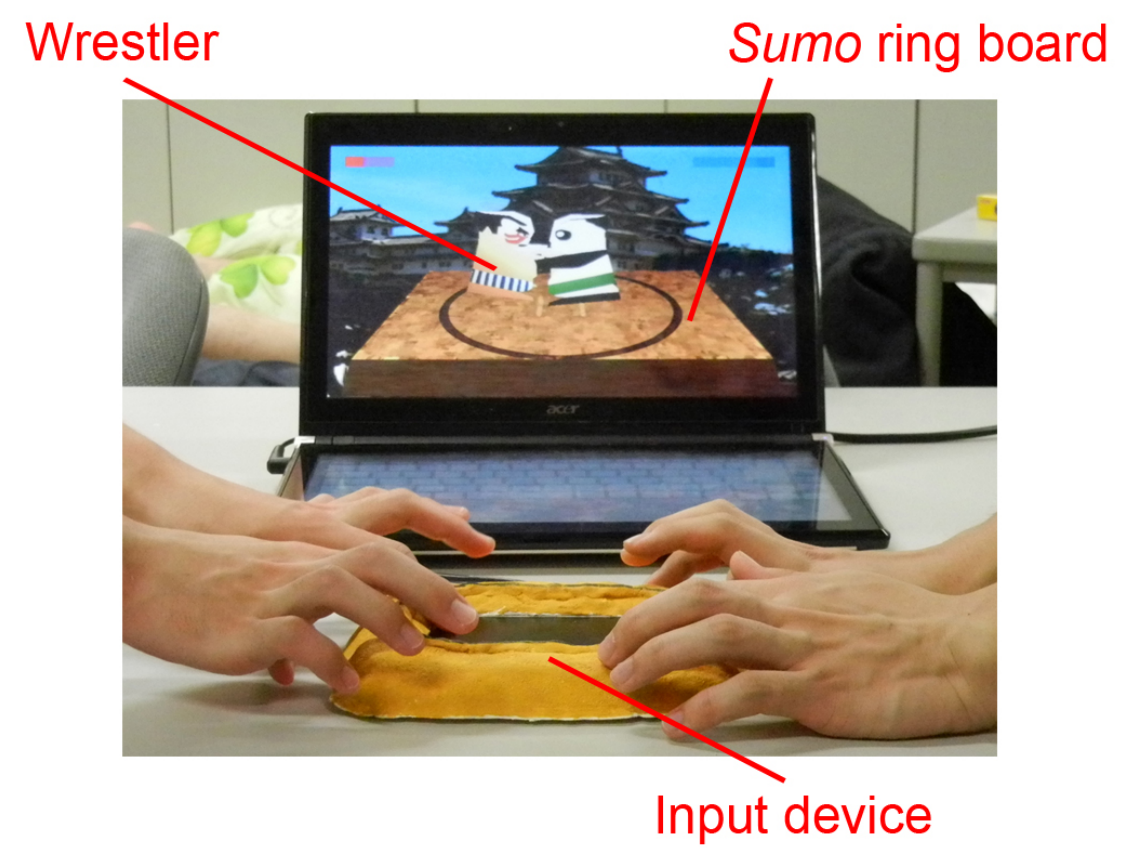

Fig. 2. Dosukoi-Tap's over view 


\subsection{The rules and strategy}

Dosukoi-Tap provides simple operation similar to the actual paper sumo. Each player chooses his/her favorite wrestler character in the selection screen and the wrestlers are arranged as facing each other on the sumo ring board on the screen. Each player lets his/her wrestler move by tapping on his/her own-side of the ForcePad as depicted in Fig.2. The wrestlers move forward and push each other in response to the repetition of tapping. Tapping affects not only wrestler's motion but also their balance. A wrestler loses when it is pushed out from the sumo ring or it loses its balance and falls down.

This game provides not only the actual paper sumo operations but also the following original operations to heat up a match:

Tsuppari. When a player does swipe toward the opponent, his/her wrestler does an offence action to push strongly. However it's easy to lose balance.

Harai. When a player does swipe toward oneself, wrestler moves back to avoid the Tsuppari of it's opponent.

In this game, the tapping and the two original operations involve players' strategic behaviors. In order to win a match, they must repeat tapping while considering wrestler's balance. Although Tsuppari is effective in pushing out an opponent, the operation carries the risk of falling down when the opponent does Harai.

\subsection{Interactive System}

In Dosukoi-Tap, we employ the ForcePad developed by Synaptics [1], as the sumo ring board. In the actual paper sumo game, a player controls his/her paper wrestler using both strength and frequency of tapping so we need the input device that can measure finger locations and their pressure strength. ForcePad is a touchpad which is able to detect multiple fingers of variable pressure. Furthermore, it is about the same size as actual paper sumo ring board so players can have similar perception as actual one as depicted in Fig.3.

Tapping operation moves the wrestler. In actual paper sumo, the motion of wrestlers is not stable because tapping operations vibrate only sumo ring board made of paper. The wrestlers fall down easily and also do not easily grapple with each other so it is difficult for a player to let his/her wrestler fight. The wrestler moves and pushes one another in response to the force of each tap. The force also affects the wrestler's center of gravity balance according to the position of each tap on the ForcePad. Players must keep the left-right balance to win a match. 

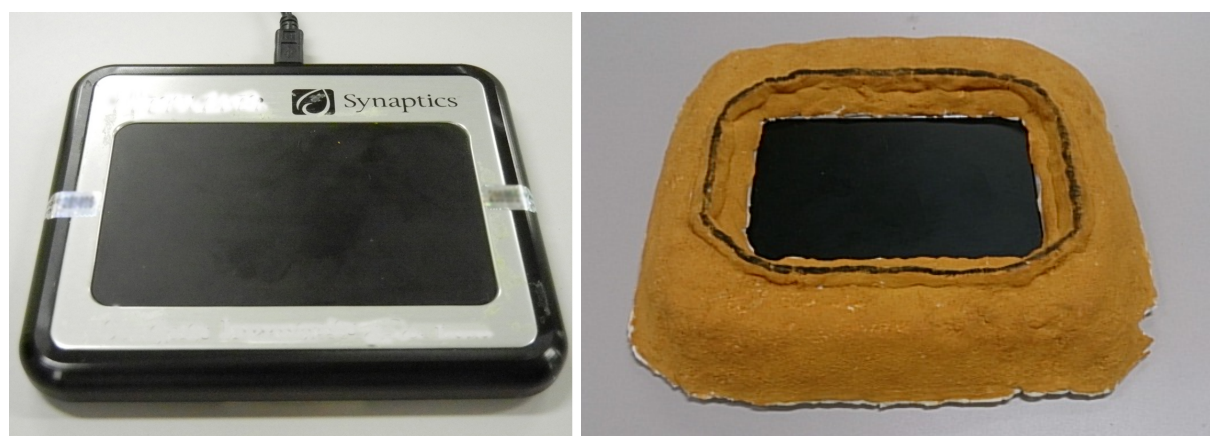

Fig. 3. ForcePad: a multi-finger tracking and pressure-sensitive device

\section{Concluding Remarks}

Dosukoi-Tap has been realized Japanese traditional game paper sumo by employing multi-finger tracking and pressure-sensitive device. This game can be played by simple tapping operation like actual paper sumo and solved some of the problems of it. The original operations have also increased the excitement of the "paper sumo". The users who know or do not know actual paper sumo rules easily understand the operation method, and the strategy is considered because of these simple operations. In recent years, many traditional games including paper sumo have not been played frequently. These games can be reborn in a more interesting game by solving some of problems and giving new technology. It will lead us to rediscovery of the fun that we had lost in these days.

\section{References}

1. ForcePad, http://www.synaptics.com/solutions/products/forcepad 